\title{
Assessment of Anemia Levels in Infants and Children in High Altitude Peru
}

\author{
Roxanne Amerson ${ }^{1}$, Lisa Miller ${ }^{1}$, Michelle Glatt ${ }^{2}$, Kate Ramsey $^{1} \&$ Jenna Baker $^{1}$ \\ ${ }^{1}$ School of Nursing, Clemson University, Clemson, SC, USA \\ ${ }^{2}$ Sacred Valley Health, Ollantaytambo, Peru \\ Correspondence: Roxanne Amerson, School of Nursing, Clemson University, Clemson, SC, USA. Tel: \\ $1-864-250-5565$.
}

\author{
Received: March 8, 2017 Accepted: April 4, 2017 Online Published: April 7, 2017 \\ doi:10.5539/gjhs.v9n7p87 \\ URL: https://doi.org/10.5539/gjhs.v9n7p87
}

\begin{abstract}
When prevalence rates of anemia exceed $40 \%$, the World Health Organization recognizes this as a severe public health problem. In Peru, approximately 43.5\% (urban) and 51.1\% (rural) of children between the ages of 6 and 36 months have anemia. Currently, limited data exists regarding prevalence rates in many of the high altitude regions of Peru. The main purpose of this pilot study was to establish evidence of anemia in infants and children ( 7 months through 5 years of age) living in the rural, mountainous region of Ollantaytambo District. This pilot study utilized a quantitative, cross-sectional design to assess the presence of anemia in infants and children. Hemoglobin levels were collected from 160 children across 12 villages where elevations ranged from 2800 to 4100 meters above sea level. Chi Square tests compared anemia with age ranges, altitude, anthropometric measures, breastfeeding patterns, and types of communities. Adjusted hemoglobin levels established $47.5 \%$ of the 160 participants were anemic. Chi Square results indicated children aged 25-36 months and children living in communities at 3100 and 4100 meters displayed higher than expected rates of anemia. Results confirmed high rates of anemia and the need for education related to dietary factors.
\end{abstract}

Keywords: anemia, global health, high altitude, iron deficiency, pediatric nursing, promotoras, Peru, screening

\section{Introduction}

In 2011, the World Health Organization (WHO) estimated that globally $43 \%$ of children experience anemia (2015). When prevalence rates of anemia exceed $40 \%$, the WHO recognizes this as a severe public health problem (WHO, 2008). The most common etiology of anemia is iron deficiency, accounting for approximately $50 \%$ of cases. Iron deficiency anemia (IDA) is associated with impaired cognitive development in children (Mujica-Coopman et al., 2015). The prevalence of anemia varies based on the resources of the country; consequently, anemia is a major health problem for children in low-income or low-resource countries. Although Peru is a high-middle income country, the Ministerio de Salud reports that approximately 43.5\% (urban) and 51.1\% (rural) of children in Peru between the ages of 6 and 36 months have anemia (2015). While the overall economic status of the country may be relatively high, many departments are impoverished with very limited resources. Depending on geographical factors, income levels, nutritional factors, and access to healthcare; the prevalence of anemia in Peru varies from a high of $76 \%$ in Puno (southeastern region along the Andes Mountain range) to a low of $34 \%$ in La Libertad (coastal region along the western border).

Limited surveillance data currently exists regarding the incidence and prevalence of anemia in the rural, less-populated, mountainous regions of Peru. The Centers for Disease Control and Prevention (CDC) Vision for Public Health Surveillance in the $21^{\text {st }}$ Century recognizes surveillance as a systematic collection of data required to identify health concerns, plan for public health interventions, and evaluate health programs (2012). An effective surveillance program requires accurate and current data before establishing a health intervention. Once data clearly identifies a need, public health professionals can begin a program to plan, implement, and evaluate disease prevention and control. The CDC Vision acknowledges the need for strengthening global health surveillance in low resource countries where surveillance programs are weak or sustainability is unstable. Global health surveillance is one of six priority areas to be addressed to move public health forward in the $21^{\text {st }}$ century.

A complex set of factors affects the incidence and prevalence of anemia. It is crucial to establish a clear presence of 
the health problem in any community before proceeding with interventions. The main purpose of this pilot study was to establish evidence of anemia in infants and children ( 7 months through 5 years of age) living in the rural, mountainous region of Ollantaytambo District within the Department of Cusco, Peru. Estimates from the Ministerio de Salud (2015) indicate the current prevalence rate at 53.1\% for children (6 months to 36 months) in this region. It is important to note that the current statistics most likely been gathered from the urban areas of this department. Recently, a lack of success in surveillance programs and low coverage rates for anemia programs in Latin America have been documented in the literature; thus substantiating the need for data gathering in the remote, high altitude communities where access to healthcare is non-existent (Mujica-Coopman et al., 2015).

Emerging research from Peru related to childhood anemia, specific to populations from the mountainous regions, is sparse. Most published studies conducted in Peru have been completed in more urban areas with infants and children, or women. Few have focused on the 6 month to 5-year age range specifically in high altitude regions. Of these studies, the overarching theme demonstrates a prevalence of IDA along with other nutritional deficits. Finkelstein, O'Brien, Abrams, and Zavaleta (2013) investigated the iron absorption rates in Peruvian breastfed infants at 2 and 5 months of age to evaluate early iron absorption from the mother during pregnancy and after birth. The results of this study found that approximately $79 \%$ of infants developed anemia by 5 months of age suggesting that infants did not have sufficient stores of iron at birth to sustain growth over the first 4-6 months of life.

Chaparro and Estrada (2012) documented the nutrition stages for the different departments of Peru. The results indicated that stunting in children less than 5 years old was highest (54.6\%) among high altitude regions and the regions with high rates of poverty. Stunting is a decrease in skeletal growth resulting in a shorter stature and is associated with nutritional deficits including IDA.

Other researchers have explored additional contributing factors, such as parasitic disease and altitude. Cabada et al. (2015) conducted a study in six villages in the Cusco Region of Peru to evaluate parasitic disease, anemia, and malnutrition in children 3-12 years of age. Over $47 \%$ of the 240 children were infected with at least one helminth or protozoa and $48 \%$ of the children were anemic. In Lima, Peru, anemic children aged 6-35 months of age were evaluated to determine the response to zinc or zinc and Vitamin A on hemoglobin values, growth rates, and the presence of diarrhea (Alarcon et al., 2004). Adding zinc to iron supplements significantly improved hemoglobin levels, yet the addition of Vitamin A did not improve the treatment significantly. Altitude also affects hemoglobin levels as the body attempts to compensate by increasing the production of red blood cells, which result from hypoxia that occurs at high altitudes. Maternal hemoglobin levels and the associated perinatal fetal outcomes were analyzed in low altitude (LA) and high altitude (HA) communities in Peru (Gonzales, Steenland, \& Tapia, 2009). This study compared women from LA and HA regions of Peru. Pregnant women at HA had higher hemoglobin values; higher rates of previous stillbirths, and small for gestational age births than women residing at LA. Therfore, the study concluded that HA was associated with reduced fetal growth.

This pilot study sought to answer the following research questions:

1). Among infants and children (7 months through 5 years of age) living in HA communities of rural Peru, what are the anemia rates in communities served by a promotora program compared to anemia rates in communities without a promotora program?

2). Do significant relationships exist between anemia and age, altitude, anthropometric measures, breastfeeding, and types of communities in HA Peru?

It was hypothesized that communities served by a promotora program would have lower rates of anemia. A promotora, also known as a community health worker, provides health education for parents of infants and children living in rural communities where access to health care is limited.

\section{Method}

A collaborative partnership with Sacred Valley Health (SVH), a non-governmental organization based in Peru, and a US-based school of nursing developed the research protocol. The staff of SVH worked with the university faculty to develop the survey instrument and to design a culturally appropriate research plan for screening children in the local communities. An independent cultural expert in Peru reviewed the plan to evaluate the cultural appropriateness prior to the institutional review. The study was approved by the institutional review board of the US-based university.

All personnel involved in the research process, including interpreters of SVH and the US-based nursing students, participated in human subjects training. The training materials for the staff and interpreters of SVH were specifically adapted to Spanish, low literacy levels, and the cultural context of Peru. Case scenarios were developed and incorporated into the training for the interpreters to help them understand the importance of 
confidentiality in the community. The US-based nursing students received training for bloodborne pathogens, collection procedures for pediatric blood specimens, and basic operation of the equipment for hemoglobin testing prior to arriving in Peru.

\subsection{Study Setting}

Sacred Valley Health currently operates in the district of Ollantaytambo, Peru. The mission of SVH is to improve the health of rural Peruvians by training promotoras in health promotion and disease prevention techniques. Promotoras are usually women from the local community who have received special training to address health issues and have the knowledge to provide culturally appropriate education and treatments (Amerson, Hall-Clifford, Thompson, \& Comninellas, 2015). During this study, promotoras provided community members with health education, information about IDA, and assured that anemic children received appropriate follow-up.

The district of Ollantaytambo is located in the heart of the Peruvian Andes, about 70 kilometers from the city of Cusco. The entire district sits above 2800 meters of elevation with many communities located above 4000 meters. At last census, the district had a population of nearly 8,950 residents, $60 \%$ of which live below the Peruvian poverty line (Instituto Nacional de Estadística e Informática, 2007) (INEI).

Typically, communities of Ollantaytambo District are geographically isolated, accessible only by primitive road or footpath with very limited cell phone service. Fewer than half of the surveyed communities have access to health facilities within a one-hour walk of their home. Family homes are most commonly single room stone structures with dirt floors. About $58 \%$ of community members speak the indigenous local language of Quechua as their only language (INEI, 2007). Food insecurity is influenced by what crops traditionally grow at these elevations. Potatoes grow readily at high elevations and are culturally the centerpiece of almost every meal, but they are not particularly high in iron. Animal-based protein is available, but not in quantities that allow for daily consumption. As a result, the Andean diet is not generally rich in micronutrients like iron.

\subsubsection{Informed Consent}

The staff and promotoras of SVH played a crucial role in the development of the consent forms. After careful review and several revisions to ensure the appropriate literacy level, an informational letter for parents was developed. A written informed consent was not feasible due to extremely low literacy levels in the communities. Instead, informational letters describing the purpose of the study and procedures were read to each parent and verbal consent was obtained prior to enrollment. The informational letters used basic terminology at a lower educational level to ensure that all participants would be able to understand the purpose, procedures, risks, benefits, and voluntary nature of the research. Interpreters read the informational letter in either Spanish or Quechua, depending on the language of the guardian. A small packet of iron-rich lentils was given to the family for each child that participated in the screening.

\subsection{Research Design}

This quantitative, cross-sectional design utilized a purposive sampling technique due to the small scale of the pilot study and the similar cultural context of families living among different villages in high altitude regions of Peru.

\subsection{Data Collection}

Infants and children were eligible for enrollment if they met the age requirement ( 7 months through 5 years of age) and had a guardian present to give consent to participate in the research project. A demographic questionnaire gathered information regarding age, previous history of anemia, current iron supplements, and dietary patterns. Parental contact information was obtained for follow-up and referral purposes in the case of a critical hemoglobin level. If a parent or child was there to receive regular care through the SVH vitamin campaign, each person was assured they would receive their regular care/education even if they chose not to participate in the research.

Length/height and weight were measured using appropriate international anthropometric equipment including a height rod and wireless scale that could be adjusted to uneven environments. Based on the obtained measurements, a risk assessment was conducted using the WHO's Child Growth Standards to plot weight-for-age and length/height-for-age with a z-score (WHO, 2006). The z-score is the number of standard deviations (SD) below the median weight or length/height. Below $-2 \mathrm{SD}$ is a commonly recognized cut-off score to indicate stunting or underweight and was used in this study to identify children with growth risks. Z-scores are preferred to track the clinical nutrition status because severely malnourished children may fall outside the standard percentile ranges for anthropometric measurements (de Onis et al., 2012).

\subsubsection{Sample Size Calculation}

A power calculation indicated a sample of approximately 162 participants was appropriate to allow for statistical 
analysis based on a $90 \%$ confidence level and a margin error of 5\%. Specimens were collected from 166 infants and children. Six questionnaires were missing data; therefore, these results were deleted from the analysis. The final sample consisted of 160 infants and children.

\subsubsection{Collection of Blood Specimens}

With faculty oversight, student nurses obtained a capillary blood sample by a finger or heel stick, depending on the weight of the infant or child. Blood specimens were analyzed using the HemoCue ${ }^{\circledR}$ and the result was read on site to provide the parent or guardian with immediate results. The hemoglobin value and the growth risk based on height and weight were documented for further analyses.

\subsubsection{Determination of Anemia}

Normal hemoglobin concentration for infants and children ages 7 months to 5 years is defined as $11.0 \mathrm{~g} / \mathrm{dL}(110 \mathrm{~g}$ per L) or higher at sea level (WHO, 2011). In the field, hemoglobin levels $<13 \mathrm{~g} / \mathrm{dl}$ in high altitude ( $>2700$ meters) are generally recognized as indicative of anemia in infants (6 months or older) and children up to 5 years of age, and $13.5 \mathrm{~g} / \mathrm{dl}$ for children 5-11 years old (Burger \& Pierre-Louis, 2002). According to the WHO guidelines, the hemoglobin value was adjusted for the high altitude of the Andes Mountains and anemia was diagnosed. Each measured hemoglobin $(\mathrm{Hgb})$ was adjusted utilizing the following formula:

$$
\text { Adjusted } \mathrm{Hgb}=\text { Unadjusted } \mathrm{Hgb}+0.32 \times(\text { altitude in meters } \times .0033)-0.22 \times(\text { altitude in meters } \times .0033)^{2}
$$

(Stevens et al., 2013). Results were verified using an online calculator for determining anemia based on adjusted hemoglobin values (http://web1.sph.emory.edu/cdckms/hbadj\%20formula\%20for\%20altitude\%202.html). The result was compared to the normal $\mathrm{Hgb}$ value of $11.0 \mathrm{~g} / \mathrm{dL}$, with values $<11 \mathrm{~g} / \mathrm{dL}$ denoting anemia. According to the WHO (2011), hemoglobin levels between 10.0-10.9 g/dL (100-109 g per L) are considered mild anemia, 7.0-9.9 g/dL (70-99 g per L) moderate, and less than $7.0 \mathrm{~g} / \mathrm{dL}(<70 \mathrm{~g}$ per $\mathrm{L})$ are severe. In order to ensure ethical standards of research and appropriate healthcare, all children determined to have anemia were given referrals to the nearest clinic for further evaluation. It is important to note that hemoglobin levels collected in this study served as a screening for anemia and did not represent a definitive diagnosis of anemia. If a child exhibited a severe level of anemia and/or displayed physical symptoms, the child was transported to the clinic by van for immediate evaluation and treatment. At the end of the screening process, a physician's assistant associated with SVH counseled the guardian on the child's results and provided education on appropriate dietary changes.

\subsection{Statistical Analysis}

Descriptive statistics were calculated for all variables and data were checked for normality. Chi square tests compared the association between the presence of anemia and age ranges, altitudes, anthropometric measurements, breastfeeding patterns, and types of communities. Data were analyzed using IBM SPSS Statistics version 23 (SPSS Inc., Chicago, IL.). All statistical tests were two-tailed and the level of significance set at 0.05 for these analyses.

\section{Results}

\subsection{Recruitment}

Participants were recruited during two campaigns in May of 2016 in remote villages at least 2800 meters above sea level. The first campaign was held in villages where SVH does not have an established presence in the community. These villages served as reference communities. The second campaign was arranged in conjunction with previously scheduled vitamin campaigns where SVH provides health education to community members. Considerable effort was made to collect data from similar communities within the SVH and reference communities.

\subsection{Demographic Characteristics}

Data were collected from 12 communities where elevation ranged from 2800 to 4100 meters above sea level. With an overall mean elevation of 3571 meters for all communities, approximately $45 \%$ of the participants lived in HA communities over 3900 meters. Because of the collaborative partnership between SVH and the research team, participants were recruited from communities where the promotoras of SVH commonly work and reference communities as well. Sixty-six percent of participants came from the communities associated with SVH and 34\% of participants came from the reference communities. Of the 12 communities, five communities are considered isolated because they are only accessible by primitive roads or footpaths and have no health care facilities. All five of the isolated communities in this study are currently served by the promotoras of SVH. A total of 160 children participated in the study. Fifty-three percent were male and $47 \%$ were female. Demographic information according to age ranges is summarized in Table 1. The highest number of participants fell within the 25-36 months age range 
$(\mathrm{n}=41)$ with the smallest number of participants ranging in age from 7-12 months $(\mathrm{n}=4)$.

Table 1. Distribution of Children by Age Ranges

\begin{tabular}{llll}
\hline Age (months) & Frequency & Percent & Cumulative Percent \\
\hline $7-12$ & 4 & 2.5 & 2.5 \\
$13-24$ & 25 & 15.6 & 18.1 \\
$25-36$ & 41 & 25.7 & 43.8 \\
$37-48$ & 32 & 20.0 & 63.8 \\
$49-60$ & 33 & 20.6 & 84.4 \\
$61-72$ & 25 & 15.6 & 100.0 \\
\hline Total & 160 & 100.0 & \\
\hline
\end{tabular}

\subsection{Hemoglobin Values and the Presence of Anemia}

After hemoglobin levels were adjusted to accommodate the high altitude levels, $47.5 \%$ of the 160 participants were found to be anemic. Overall, adjusted hemoglobin levels ranged from 7.0 to $15.5 \mathrm{~g} / \mathrm{dl}$ with a mean of $11.07 \pm$ SD $1.54 \mathrm{~g} / \mathrm{dl}$. It is important to note that SVH communities tend to be located in higher elevations between 3600-4100 meters, although an effort was made to recruit participants from various communities where elevations ranged from 2800-3900 meters. Adjusted hemoglobin values ranged from 7.8 to $14.0 \mathrm{~g} / \mathrm{dl}$ with a mean of $11.20 \pm$ SD $1.28 \mathrm{~g} / \mathrm{dl}$ in SVH communities and 7.0 to $15.5 \mathrm{~g} / \mathrm{dl}$ with a mean of $1.94 \pm \mathrm{SD} 1.28 \mathrm{~g} / \mathrm{dl}$ in the reference communities. The rate of anemia in SVH communities was $45.7 \%$ compared to a rate of $50.9 \%$ in the reference communities.

\subsection{Past Anemia Treatment and Anthropometric Measures}

Approximately $9 \%$ of participants had received past treatment for anemia and $61.9 \%$ of children were currently taking an iron supplement or vitamin based on parental report. An interesting note, $16.4 \%$ of reference community participants reported past treatment for anemia compared to only $4.8 \%$ of SVH participants. Based on WHO Child Growth Standards for age, anthropometric measurements revealed $57.5 \%$ of participants at normal length/height and weight, with $36.9 \%$ below normal length/height, and the remaining $5.6 \%$ below in both length/height and weight.

\subsection{Breastfeeding Patterns}

In the 13-24 month age range, only $76 \%$ were reported to receive breastmilk along with complementary food. Among children identified as anemic, only $66.7 \%$ of participants age $13-24$ months were being breastfeed along with complementary food. Of children with normal hemoglobin levels, $90 \%$ of children in this age range were being breastfeed along with complementary food.

\subsection{Inferential Statistics}

Chi Square tests of independence were calculated comparing the presence of anemia and several variables; including age ranges, altitude, anthropometric measures, breastfeeding patterns, and types of communities. The Chi Square results are summarized in Table 2. No significant associations were found among anemia and types of communities, anthropometric measurements, or breastfeeding with complementary food in this sample. Significant associations were found related to anemia with age ranges and the altitude of the community. Positive adjusted residual values for the Chi Square indicated higher than expected rates of anemia in HA communities, while negative adjusted residuals indicated lower than expected rates of anemia in at least one LA community. A residual is the difference between the observed and expected values for cells of the Chi Square test. 
Table 2. Chi Square Evaluation of Anemia According to Variables

\begin{tabular}{|c|c|c|}
\hline Variable & Result & Interpretation \\
\hline Age Ranges 13-72 months & $\mathrm{x}^{2}(4)=9.7, \mathrm{p}<.05$ & $\begin{array}{l}\text { Children 25-36 months of age had higher } \\
\text { than expected cases of anemia. }\end{array}$ \\
\hline Altitude & $x^{2}(5)=17.503, p<.05$ & $\begin{array}{l}\text { Higher than expected cases of anemia at } \\
3100 \text { and } 4100 \text { meters, lower than } \\
\text { expected cases at } 2800 \text { meters. }\end{array}$ \\
\hline Type of community (reference, participating) & $\mathrm{x}^{2}(1)=.391, \mathrm{p}>.05$ & No significant association. \\
\hline Below normal height/weight & $\mathrm{x}^{2}(2)=.953, \mathrm{p}>.05$ & No significant association. \\
\hline Breastfeeding & $x^{2}(2)=2.546, p>.05$ & No significant association. \\
\hline $\begin{array}{l}\text { Age range } 7-24 \text { months and breastfeeding or } \\
\text { complementary food }\end{array}$ & $x^{2}(1)=1.167, p>.05$ & No significant association. \\
\hline
\end{tabular}

\section{Discussion}

The research team sought to determine the current rates of anemia in the underserved regions of Ollantaytambo, Peru. In this pilot study, $47.5 \%$ of the 160 children were anemic. These findings are consistent with childhood anemia rates of $48.8 \%$ in 12 villages within the region of Cusco (Cabada et al., 2015). According to a systematic review of the prevalence of anemia in Latin America from 2014, anemia is a moderate public health problem in Peru (Mujico-Coopman et al., 2015). Yet, according to the WHO guidelines when anemia exceeds $40 \%$ it becomes a severe public health problem. The results of this pilot study clearly demonstrate that anemia is indeed a severe public health concern in need of interventions in this isolated region.

Initially, it was hypothesized that in communities where promotoras conducted health education the rates of anemia would be less. Although not statisticually significant, results did demonstrate that the rate of anemia was slightly lower in the SVH communities compared to reference communities. This is thought provoking considering that the majority of SVH communities are located in HA, isolated communities. Children, who live in communities served by SVH promotoras, are monitored and parents receive teaching about anemia, risk factors, and nutrition. It is particularly relevant that over $16 \%$ of children in the reference communities had received previous treatment for anemia compared to only $4.8 \%$ of children from SVH communities. This lack of previous treatment may be reflective of the isolation and lack of access to healthcare that is common for the HA communities covered by SVH promotoras. Even though, children are more isolated and have less access to care in SVH communities, the rates of anemia are slightly lower than in the reference communities.

Additionally, the research team anticipated that high elevation would contribute to higher rates of anemia in these rural communities. In fact, the comparison of community altitudes demonstrated a statistically significant difference. The adjusted residuals of the Chi Square indicated that communities with altitudes of 3100 and 4100 meters had higher than the expected cases of anemia. Yet, only one SVH community at 4100 meters demonstrated a significantly higher than expected number of anemia cases. Further studies need to tease out which factors are contributing to the higher than expected cases in these specific communities.

The findings from this pilot study demonstrate a higher rate of anemia in the 25-36 month age range. Cabada et al. (2015) also found $77.1 \%$ of children 3-4 years of age were anemic in the Cusco Region. Toddlers aged 12-36 months of age are higher risk for IDA due to the full transition to table foods, the potential for pica concerns, and the introduction of whole cow's milk (Paoletti, Bogen, \& Ritchey, 2014). Cow's milk is low in iron, contributes to occult gastrointestinal blood loss, and may inhibit the absorption of calcium and casein. Pediatric care experts recommend screening for anemia begin by approximately one year of age. The CDC further recommends additional screening annually for ages $2-5$ years.

Anthropometric measurements indicated approximately $37 \%$ of children were below normal length/height-for-age (stunted) according to WHO standards. Less than 6\% of children showed evidence of both below average length/height- and weight-for-age. Food insecurity in the impoverished communities frequently leads to malnutrition and stunted growth. Cabada et al. (2015) noted stunting at $31.3 \%$ with $10 \%$ of children significantly underweight in the Cusco Region, but noted that the presence of stunting varied greatly based on the specific community. The higher number of children with stunting in the Ollantaytambo District appears to suggest that children are receiving sufficient calories to sustain weight, but an inadequate intake of protein leading to long term 
or past growth failure. Stunting is commonly associated with poor economic conditions where food supplies are limited in quantity or nutritional value (WHO, 2006).

Recent publications on the prevalence of anemia indicate that Peruvian children are receiving daily or weekly doses of vitamins (Mujica-Coopman et al., 2015). Yet published information indicates that iron supplementation programs cover only $12 \%$ of children in Peru. Children in HA communities are less likely to receive the benefits of a governmental iron supplementation program. Also, future vitamin campaigns should educate parents to begin iron supplementation at 4 months of age (Kohli-Kumar, 2001). Introduction of iron at this age is supported by a recent study (Finkelstein et al., 2013) in Peru where 79\% of infants developed anemia by 5 months of age.

While not statistically significant, the children in the SVH communities appear to have improved health status, in spite of the isolation, high altitude, and poverty. It is hypothesized that the improved health status is the result of the parental teaching that has been conducted in these communities since the inception of SVH's promotora program. The promotoras routinely teach parents about nutrition, parasitic infections, hygiene practices, and distribute multivitamins that contain iron. Children in the isolated communities are also more likely to suffer from parasitic diseases where hygiene practices are less than adequate. Few homes in the HA communities have indoor plumbing. In fact, $85.7 \%$ of children in SVH communities reported taking vitamin supplements at the time of the study compared to $16.4 \%$ in reference communities. It seems plausible that these education interventions are having an impact on the health of families in the communities served by $\mathrm{SVH}$.

One area that bears further investigation is breastfeeding. Over a decade ago, international recommendations from the WHO provided public health guidelines to promote breastfeeding until 24 months of age in developing countries where economic constraints exist (Lauer, Betran, Victora, de Onis, \& Barros, 2004). In this study almost $25 \%$ of the children screened, under the age of 2 years, were not receiving breastmilk. Of the anemic children (1324 months), $33 \%$ did not receive breastmilk along with complementary food. When compared with non-anemic children, only $10 \%$ did not receive breastmilk. Unfortunately, with a total sample of only 29 children in the relevant age groups (7-12 months, 13-24 months), no statistically significant results could be obtained. The research team strongly urges further investigation to evaluate cultural beliefs about breastfeeding and time constraints (i.e., mothers working in the fields, caring for younger children) which may be contributing to early discontinuation of breastfeeding. A programming emphasis on consistent breastfeeding and complementary feeding through 24 months of age is needed with future impact evaluation.

The isolation of the mountainous communities surveyed in this pilot study has resulted in the area receiving little or no healthcare services. The research team sought to bring attention to a region where surveillance efforts for anemia are lacking. Obviously, limited generalization can be assumed from the data based on a small convenience sample of 160 children. The research team fully acknowledges the limitations of the sample sizes. Small sample sizes influenced the ability to obtain statistically significant results when comparing anemia with factors such as anthropometric measures and breastfeeding patterns. Testing in this region where few children have ever been screened for anemia was valuable and has laid the groundwork for future screenings and education programs to combat the high levels of anemia that exist. Sacred Valley Health has revised educational materials and plans to implement additional programs in the local communities in the near future based on these findings.

\section{Conclusion}

Anemia is a multifactorial event affected by unsanitary conditions, helminth infections, and consuming foods low in iron. The promotora program of SVH provides education related to handwashing, sanitation, food and water preparation; and conducts vitamin campaigns. A referral protocol is currently in place for anemic children. Future recommendations for these communities should include continued anemia screenings, assessment of breastfeeding routines, identification of risk factors related to pica, education regarding the introduction of cow's milk post-breastfeeding, and continuation of deworming protocols as appropriate. The results from this anemia screening confirmed the presence of significantly high levels of anemia in children in the remote, HA regions of Peru. These findings are consistent with the reported prevalence rate of 51.5\% in rural Peru (Ministerio de Salud, 2015). Obviously, the high rates of anemia in this region need to be addressed with culturally appropriate interventions. Communities, where promotoras are currently working to provide case management and education, appear to be faring marginally better with slightly lower rates of anemia and improved adherence to vitamin supplementation. With this data, the research team plans to seek funding to further the study, reach larger populations, and investigate the impact of nutritional interventions in the future. Anemia is a condition that affects millions of children around the world. Finding ways to make a positive impact on anemia at the local level will ultimately improve health outcomes for children at the global level. 


\section{Acknowledgements}

This study was funded by the Mary Lohr Endowment through the Clemson University School of Nursing (grant number 2280096). The research team gratefully acknowledges the collaborative partnership and the staff of Sacred Valley Health of Ollantaytambo, Peru, for their assistance in the community, Dr. Veronica Parker for her assistance with statistical analysis, and the nursing students of the Global Health Certificate Program at Clemson University.

\section{Competing Interests Statement}

The authors declare no conflicts of interest.

\section{Authors' Contributions}

All authors participated in the collection of data, except JB. All authors assisted in the writing of the manuscript and reviewed the final submission.

\section{References}

Alarcon, K., Kolsteren, P., Prada, A., Chian, Velarde, R., Recho, I., \& Hoeree, T. (2004). Effects of separate delivery of zinc or zinc and vitamin A on hemoglobin response, growth, and diarrhea in young Peruvian children receiving iron therapy for anemia. American Society for Clinical Nutrition, 80, 1276-1282.

Amerson, R., Hall-Clifford, R., Thompson, B., \& Comninellas, N. (2015). Implementation of a training program for low literacy promotoras in oral rehydration therapy. Public Health Nursing, 32(2), 177-185. https://doi.org/10.1111/phn.12152

Burger, S., \& Pierre-Louis, J. N. (2002). How to assess iron deficiency anemia and use the Hemocue? Helen Keller International. Retrieved from http://pdf.usaid.gov/pdf_docs/Pnacw824.pdf

Cabada, M., Goodrich, M., Graham, B., Villanueva-Meyer, P., Deichsel, E., Lopez, M. ... White, A. (2015) .Prevalence of intestinal helminths, anemia, and malnutrition in Paucartambo, Peru Revista. Panamericana de Salud Publica, 37(2), 69-75.

Centers for Disease Control and Prevention (CDC). (2012). CDC's Vision for Public Health Surveillance in the 21st Century. Retrieved from https://www.cdc.gov/mmwr/pdf/other/su6103.pdf

Chaparro, M. P., \& Estrada, L. (2012). Mapping the nutrition transition in Peru: Evidence for decentralized nutrition policies. Rev Panam Salud Publica, 32(3), 241-244. https://doi.org/10.1590/S1020-49892012000900010

de Onis, M., Onyango, A., Borghi, E., Siyami, A., Blossner, M., \& Lutter, C. (2012). Worldwide implementation of the WHO Child Growth Standards. Public Health Nutrition, 1-8. https://doi.org/10.1017/S136898001200105X

Finkelstein, J. L., O'Brien, K. O., Abrams, S. A., \& Zavaleta, N. (2013). Infant iron status affects iron absorption in Peruvian breastfed infants at 2 and 5 mo of age. American Journal of Clinical Nutrition, 98(6), 1475-1484. https://doi.org/10.3945/ajcn.112.056945

Gonzales, G. F., Steenland, K., \& Tapia, V. (2009). Maternal hemoglobin level and fetal outcome at low and high altitudes. American Journal of Physiology, 297(5), R1477-R1485. https://doi.org/10.1152/ajpregu.00275.2009

Instituto Nacional de Estadística e Informática. (2007). Censos Nacionales 2007 XI de Populación de Vivienda. Retrieved from http://censos.inei.gob.pe/Censos2007/redatam

Kohli-Kumar, M. (2001). Screening of anemia in children: AAP recommendations - A critique. Pediatrics, 108(3), e56. https://doi.org/10.1542/peds.108.3.e56

Lauer, J., Betran, A.P., Victora, C., de Onis, M., \& Barros, A. (2004). Breastfeeding patterns and exposure to suboptimal breastfeeding among children in developing countries: review and analysis of nationally representative surveys. BMC Medicine, 2(26), 1-29. https://doi.org/10.1186/1741-7015-2-26

Ministerio de Salud. (2015). PERU: Proporción de ni-os de 6 a menos de 36 meses de edad con anemia por departamento. Retrieved from http://www.app.minsa.gob.pe/bsc/Detalle_IndBSC.asp?lcind=8\&lcobj= $2 \&$ lcper $=1 \&$ lcfreg $=7 / 4 / 2016$

Mujica-Coopman, M. F., Brito, A., López de Romañana, D., Ríos-Castillo, I., Cori, H., \& Olivares, M. (2015). Prevalence of anemia in Latin American and the Caribbean. Food and Nutrition Bulletin, 36(Supplement2), S119-S128. https://doi.org/10.1177/0379572115585775 
Paoletti, G., Bogen, D., \& Ritchey, A. (2014). Severe iron-deficiency anemia still an issue in toddlers. Clinical Pediatrics, 53(14), 1352-1358. https://doi.org/10.1177/0009922814540990

Stevens, G.A, Finucane, M.M., De-Regil, L.M., Paciolek, C.J., Flaxman, S.R., Branca, F., ... Ezzati, M. (2013). Global, regional, and national trends in haemoglobin concentration and prevalence of total and severe anaemia in children and pregnant and non-pregnant women for 1995-2011: a systematic analysis of population-representative data. Lancet Global Health, 1(1), e16-25. https://doi.org/10.1016/S2214109X(13)70001-9

World Health Organization. (2006). WHO Child Growth Standards: Length/Height-for-Age, Weight-for-Age, Weight-for-Length, Weight-for-Height and Body Mass Index-for-Age: Methods and Development. Geneva: World Health Organization. Retrieved from http://www.who.int/childgrowth/standards/technical_report/en/

World Health Organization. (2008). Worldwide Prevalence of Anaemia 1993-2005: WHO Global Database on Anaemia. Retrieved from http://apps.who.int/iris/bitstream/10665/43894/1/9789241596657_eng.pdf

World Health Organization. (2011). Haemoglobin concentrations for the diagnosis of anaemia and assessment of severity. Geneva: World Health Organization. Retrieved from http://www.who.int/vmnis/indicators/haemoglobin.pdf

World Health Organization. (2015). The global prevalence of anaemia in 2011. Geneva: World Health Organization. Retrieved from http://apps.who.int/iris/bitstream/10665/177094/1/9789241564960_ eng.pdf?ua $=1 \& u a=1$

\section{Copyrights}

Copyright for this article is retained by the author(s), with first publication rights granted to the journal.

This is an open-access article distributed under the terms and conditions of the Creative Commons Attribution license (http://creativecommons.org/licenses/by/4.0/). 